\title{
Strain Measurement With Nanometre Resolution By Transmission Electron Microscopy.
}

\author{
David Cooper ${ }^{1, a^{*}}$ and Jean-Luc Rouviere ${ }^{2, b}$ \\ ${ }^{1}$ CEA-LETI, MINATEC Campus, 17 rue des Martyrs, 38054 Grenoble Cedex 9, France. \\ ${ }^{2}$ CEA-LETI, MINATEC Campus, 17 rue des Martyrs, 38054 Grenoble Cedex 9, France. \\ adavid.cooper@cea.fr, bjean-luc.rouviere@cea.fr,
}

Keywords: Strain mapping, Dark Holography, Nanobeam Diffraction, Geometrical Phase Analysis

\begin{abstract}
Strain is routinely used in state-of-the-art semiconductor devices in order to improve their electrical performance. Here we present experimental strain measurements obtained by different transmission electron microscopy (TEM) based techniques. Dark field electron holography, nanobeam electron diffraction (NBED) and high angle annular dark field scanning electron microscopy (HAADF STEM) are demonstrated. In this paper we demonstrate the spatial resolution and sensitivity of these different techniques on a simple calibration specimen where the accuracry of the measurement can easily be assessed.
\end{abstract}

\section{Introduction.}

Although the effects of introducing strain in semiconductor devices is well known from an electrical point of view, little is known about the distribution of strain. Indeed, it is only in the last few years that methods that can measure the strain with the required level of spatial resolution have been developed. Dark field electron holography [1], NBED [2] and HAADF STEM [3] are different TEM based techniques that have been developed in order to solve this problem. A calibration specimen was designed so that the different strain mapping techniques could be tested and compared to accurate simulations that would account for the relaxation of the thin TEM specimen. The calibration specimen that was examined was grown by reduced pressure chemical vapour deposition and comprised from top to bottom, a 150-nm-thick capping layer, then four 10nm-thick SiGe layers with Ge concentrations of $45 \%, 38 \%, 31 \%$ and $20 \%$, each separated by $30 \mathrm{~nm}$ of silicon on a silicon substrate. As the growth is epitaxial, the lattice spacing in the in-plane (xdirection) does not change. However, in the growth (z-direction) the lattice parameter is expanded relative to the unstrained substrate due to the presence of $\mathrm{Ge}$ and it is this relative expansion which is measured. The dark holography experiments were performed using a probe corrected FEI Titan operated at $200 \mathrm{kV}$, the precession NBED (PED) and the HAADF STEM experiments were performed using a double corrected FEI Titan operated at $200 \mathrm{kV}$. All of the data was processed using software written at CEA.

\section{Dark Field Electron holography}

Figure 1 shows the process flow for dark field electron holography. An electron biprism is used to interfere an electron wave that has passed through a strained region of interest with an electron wave that has passed through an unstrained reference, such as the substrate. By using an objective aperture to select a diffracted beam corresponding to the lattice places of interest, a phase image can be calculated by Geometrical Phase Analysis (GPA) that corresponds to a displacement field for the chosen set of lattice parameters from which a strain map can be calculated. Dark field holography is well adapted for the semiconductor industry as 2D strain maps can rapidly be produced with a field of view of up to a micron and a spatial resolution of $4 \mathrm{~nm}$. We have benefited here from the stability of the Titan TEM to acquire electron holograms for up to one minute to provide strain maps with a sensitivity of $+/-0.02 \%$ [4]. 


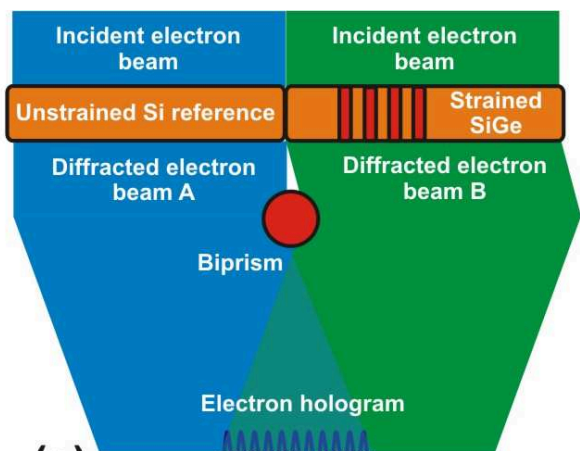

(a)

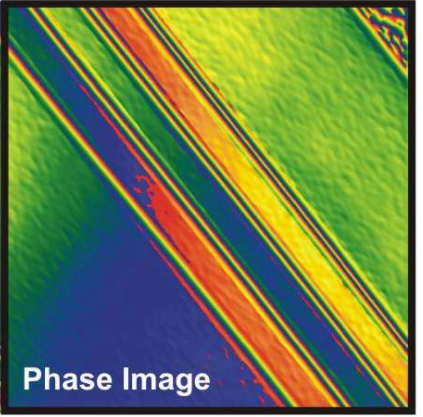

(d)

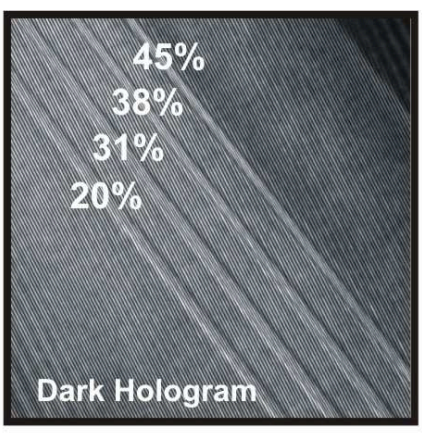

(b)

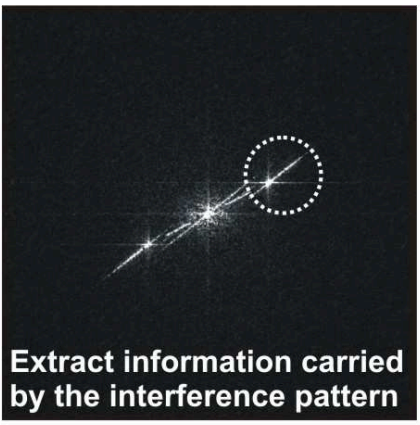

(c)

Figure 1(a) Schematic of the optical setup used in dark field holography. (b) A $\{004\}$ dark field electron hologram of a Si calibration specimen containing 10-nm-thick SiGe layers with different Ge concentrations. (c) A Fourier transform of the hologram is performed and the sideband is selected from which (d) a phase image can be reconstructed. GPA is used to calculate a (e) strain map showing the SiGe strain in the $\{004\}$ growth direction. (f) A profile of the experimental results can be compared to finite element strain simulations demonstrating that this technique is fully quantitative.

\section{Nanobeam Diffraction}

Figure 2 shows the process for nanobeam electron diffraction. A near-parallel electron beam is used to provide diffraction patterns from which the local strain can be determined from the shifts in the diffraction spots relative to a reference pattern. By using the three condenser lens system in the FEI Titan TEM we can now provide an electron beam with a convergence angle of 0.2 mrads and a FWHM of $6 \mathrm{~nm}$ for $200 \mathrm{kV}$ electrons. The electron beam is scanned across the region of interest in STEM mode and the patterns are automatically processed using an in-house software to provide strain profiles. This technique is extremely easy to perform and 1D strain profiles with a sensitivity of $0.06 \%$ has now been demonstrated with a spatial resolution of $3 \mathrm{~nm}$ [5]. The main problem with NBED is that the probe is not perfectly parallel, thus the diffraction pattern is composed of discs containing dynamical effects leading to incorrect measurements of the strain. Recently we have shown that by precessing the beam, evenly illuminated diffraction spots can be recorded which leads to accurate strain maps. In addition, a more convergent beam can be used leading to an improved spatial resolution or around $2 \mathrm{~nm}$ and a sensitivity of $0.02 \%$ [6]. Figure 3 shows results obtained using precession diffraction, more than 1500 diffraction patterns have been acquired in order to calculate this strain map (a) a pseudo HAADF image is formed from the diffraction patterns showing the region examined and (b) a strain map for the $\{004\}$ direction. Figure 3(c) shows a strain profile extracted from an averaged area of $10 \mathrm{~nm}$ compared to simulations demonstrating the excellent signal to noise obtained. The results from the precession are slightly less than the simulations as a thinner specimen was examined than for the dark holography results. 

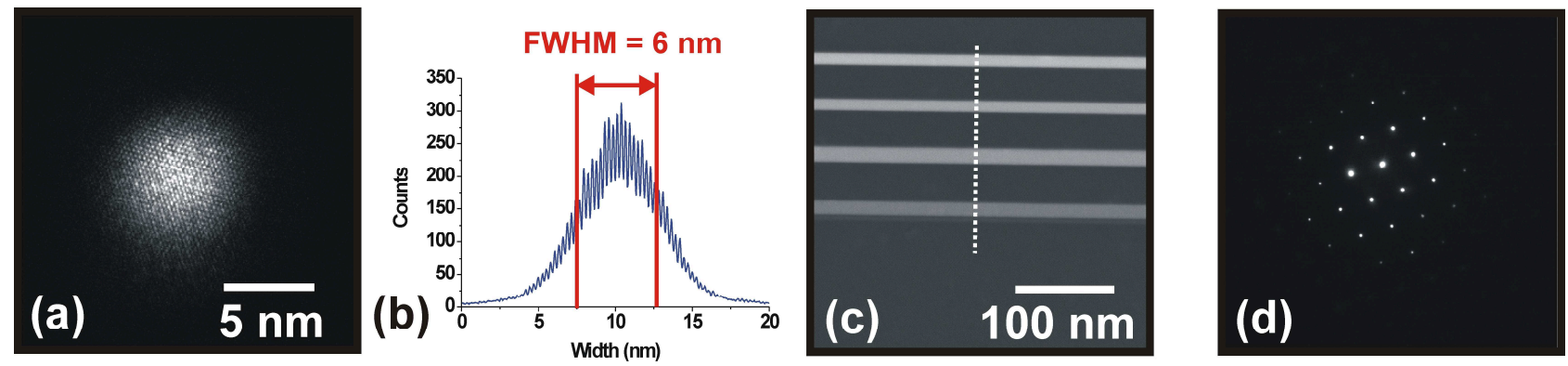

Figure 2 Schematic of the basic NBED procedure. (a) A $200 \mathrm{kV}$ near-parallel electron probe on a $\{110\}$ Si TEM specimen (b) The FWHM here is $6 \mathrm{~nm}$. (c) The electron beam is scanned across the specimen in STEM mode and a diffraction pattern is recorded at each point from which the strain can be determined from the shift in the spots automatically using a peak fitting software developed at CEA/INAC.
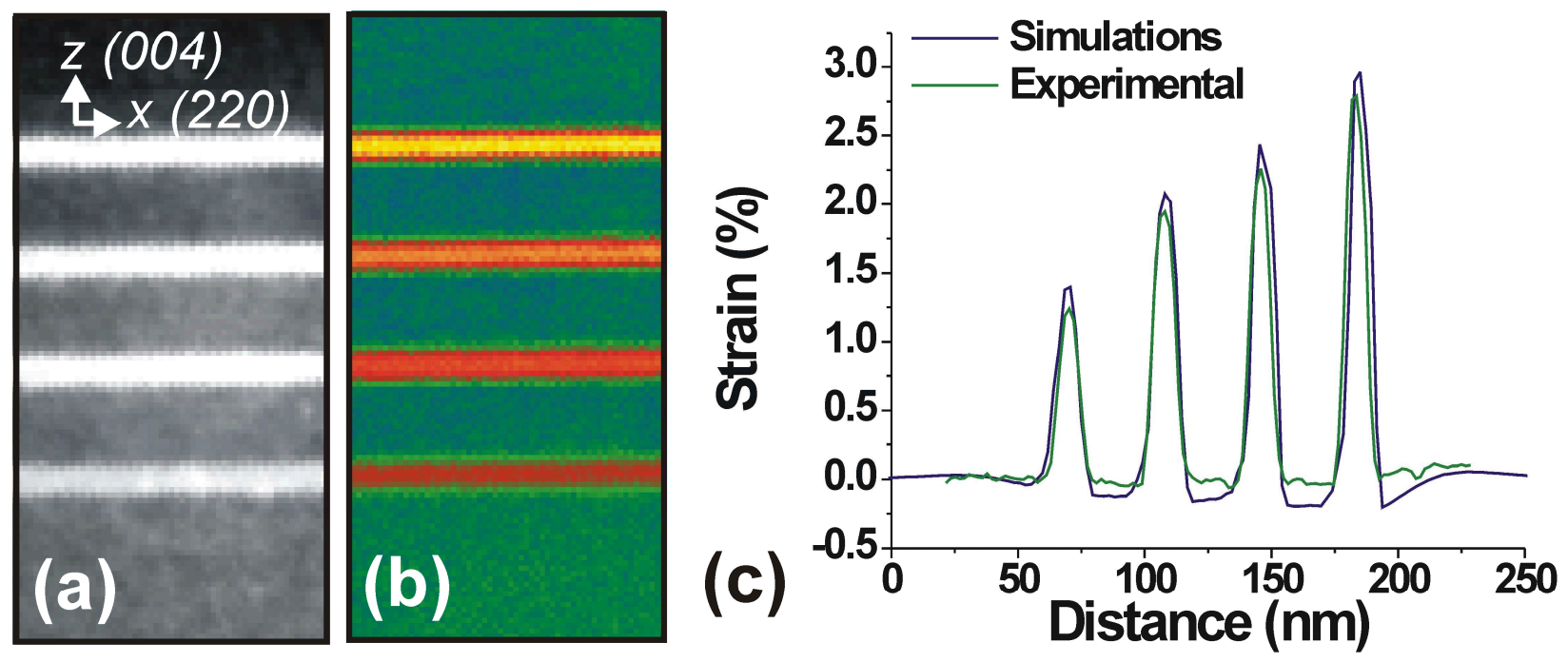

\section{$20 \mathrm{~nm} \quad-1 \% \quad 0 \% \quad 1 \% \quad 2 \%$}

Figure 3 (a) Pseudo HAADF Image of calibration specimen formed from the diffraction patterns (b) strain map for the $\{004\}$ growth direction (c) experimentally obtained strain profile compared to simulations.

\section{Geometrical Phase Analysis}

Strain maps have been obtained from high resolution transmission electron microscopy images for many years, however the interpretation of TEM images is complicated. The contrast is dependent on specimen thickness and focus, thus the results can be misleading unless a very small field of view is examined which is not always suitable for semiconductors. The excellent stability of the latest generation electron microscopes means that strain maps can now be obtained directly from HAADF STEM images [7]. Figure 4 shows the process for forming strain maps by HAADF STEM. A HAADF STEM Image is acquired and a FFT is applied in order to obtain information about the components of the lattice in the direction of strain that is being examined. Here we select the information from two different and orthagonal $\{111\}$ spots as their intensity is relatively high and then two different phase images are obtained from which strain maps for the growth and in plane directions can similtaniously be obtained. In order to minimise the artefacts from the scanning, the scan direction is chosen to be in the same drection as the strain field being measured. Vertical artefacts can be seen in the strain map due to electrical interference in the scan which is not observable in the raw HAADF images. Spatial resolutions of $1 \mathrm{~nm}$ can be obtained using this technique which is relevant for many different types of specimens such as quantum dots and state of the art semiconductor devices which are currently in development [8]. 

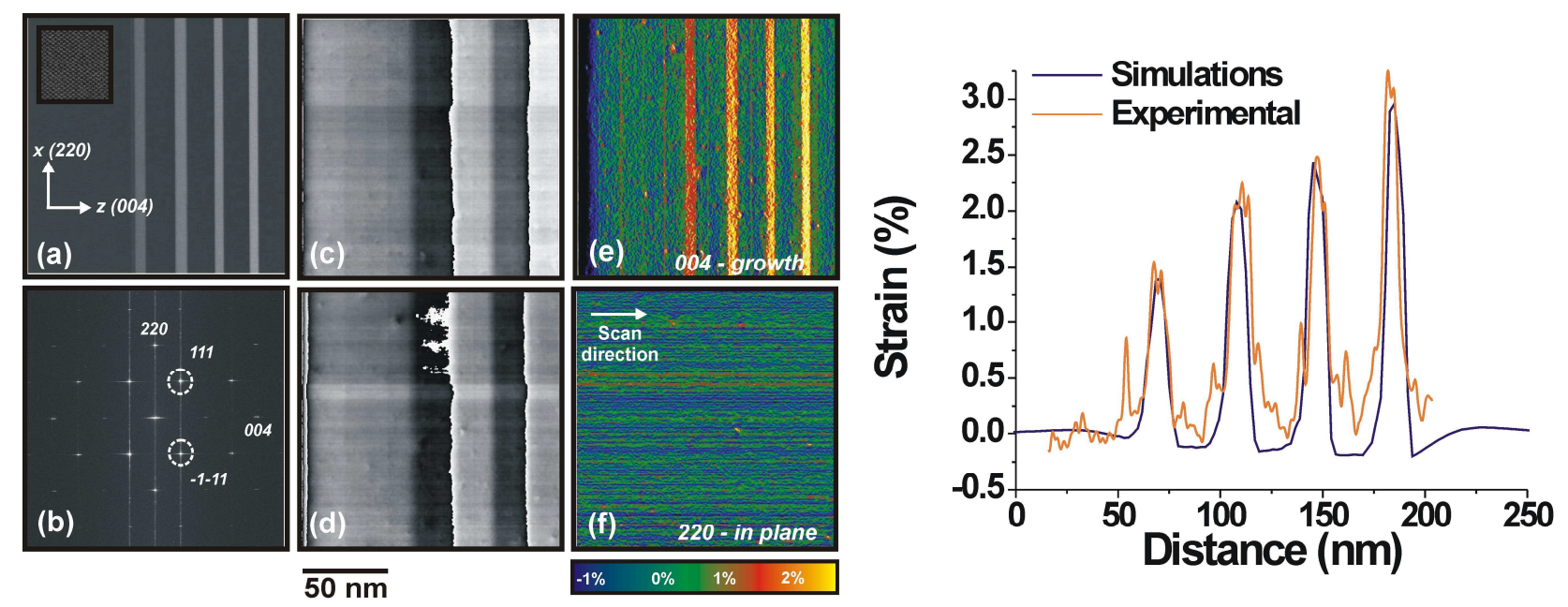

Figure 4 (a) A HAADF STEM image is acquired with the scan in the direction of the strain being measured. (b) A FFT is applied and the spots of interest are selected in order to form (c-d) two different phase images from which the strain in the growth (e) and (f) in plane can be measured. The experimentally obtained strain profile is compared to simulations, and although the accuracy is good the profile is relatively noisy.

\section{Comparison of the techniques.}

In the last few years a great deal of attention has been made on the subject of strain mapping using transmission electron microscopy and encouraging progress has been made. Dark field electron holography is an excellent technique that provides strain maps quickly with minimal data processing. However, a perfect crystal is required with a reference which is exacly aligned with the region of interest. An additional problem with dark holography is that a perfect specimen is required to prevent unwanted diffraction effects. NBED has been used mainly by the semiconductor industry as it is both easily accessible and straightforward to perform. NBED can give inaccurate results and tends only to supply strain profiles rather than maps. The application of precession to NBED now removes many of these artefacts and recent improvements in data handling means that maps can now be easily acquired, although this can be time consuming. The strain map shown in Figure 3 took one hour to acquire and an additional hour to process after the microscope was set up This can be compared to dark holography which takes less than a minute to acquire and process the data. The advantage of PED is that it is very versitile and allows strain maps to be acquired of almost any type of material. HAADF STEM is a very useful strain mapping technique which is used in the semiconductor industry as the dimensions of devices can now be less than $10 \mathrm{~nm}$, however the signal to noise limits the technique and this is used often to see if a device is "strained or not". HAADF STEM strain mapping is very insensitive to "bad specimen preparation" and is thus a robust technique. However, a very stable electron microscope is required to have acceptable signal to noise in the calculated strain maps.

\section{Summary}

There are many different methods of measureing strain with nm-scale resulution using transmission electron microscopy. These methods are used routinely in the semiconductor industry to provide important information about the processes that are used in building prototype devices. All of these different approaches have advantages and disadvantages. Figure 5 shows a summary of the different techniques in March 2014. However, the continuous developments driven by improvements in hardware, software and specimen preparation mean that the story of strain mapping in the TEM is far from over. 
Dark field holography

Excellent sensitivity $+/-0.02 \%$ demonstrated :-

1 - $10 \mathrm{~nm}$ spatial resolution $:-$

Provides a strain map instantly :-

Needs a reference region near the region of interest :

Need perfect crystals $:$

Specimen tilts limit spatial resolution $:$

Very enjoyable to perform :-)

Nanobeam diffraction with precession

Sensitivity $+/-0.02 \%$ demonstrated (-)

$1-2 \mathrm{~nm}$ spatial resolution ()

Extremely versatile (:)

Maps can now be easily acquired

Reference can be taken from anywhere :-

Boring to perform and data processing is long $(-)$

HAADF STEM strain mapping

Sensitivity $+/-0.15 \%$ demonstrated (-)

1 - $5 \mathrm{~nm}$ spatial resolution (:)

Needs a reference region near the region of interest $:$

Reasonably enjoyable to perform (-)

Insensitive to bad specimen preparation (:)

Structural information such as the polarization can be determined (-)
A biprism is required and also a stable microscope for the best sensitivity.

Best for precise measurement of strain in perfect crystals grown by epitaxy.

Precession diffraction is necessary which does not commercially available in Titans microscopes at this time.

Today seems the best technique for strain mapping.

A very stable microscope is required.

Good for rapid strain measurements if good sensitivity is not required.

Figure 5. Summary of the strengths and weaknesses of the different strain mapping techniques discussed here.

Acknowledgements. This work was partially supported by the French National Research Agency (ANR) through the RTB Program and within the IBM - ST Microelectronics - LETI alliance. DC thanks the European Research Council for the starting grant "Holoview".

\section{References}

[1] M. Hytch et al, Nanoscale holographic interferometry for strain measurements in electronic devices. Nature 453 (2008) 1086

[2] K. Usada et al, Strain characterization in SOI and strained-Si on SGOI MOSFET channel using nanobeam electron diffraction (NBD). Mat. Sci. Eng. B. 124 (2005) 143

[3] M. Hytch et al, Measurement of the displacement field of dislocations to $0.03 \AA$ by electron microscopy. Ultramicroscopy 74 (1998) 131

[4] D. Cooper et al, Dark field electron holography for quantitative strain measurements with nanometer-scale spatial resolution. Appl. Phys. Lett., 95 (2009) 053501

[5] A. Béché et al, Improved precision in strain measurement using nanobeam electron diffraction. Appl. Phys. Lett., 95 (2009) 123114

[6] J-L. Rouviere et al, Improved strain precision with high spatial resolution using nanobeam precession electron diffraction. Appl. Phys. Lett.,103, (2013) 241913

[7] D. Cooper et al, Strain mapping with nm-scale resolution for the silicon-on-insulator generation of semiconductor devices by advanced electron microscopy. J. Appl. Phys. 112 (2012) 124505

[8] D. Cooper et al, Quantitative strain mapping of InAs/InP quantum dots with $1 \mathrm{~nm}$ spatial resolution using dark field electron holography. Appl. Phys. Lett., Applied Physics Letters 99 (2011) 261911 